\title{
Informe. La red académica Information Schools: orígenes, evolución y futuribles
}

\author{
Report. The academic Information Schools network: Origins, \\ evolution and future
}

Josep Cobarsí-Morales

Cobarsí-Morales, Josep (2018). "Informe. La red académica Information Schools: orígenes, evolución y futuribles". Anuario ThinkEPI, v. 12, pp. 36-47.

https://doi.org/10.3145/thinkepi.2018.03

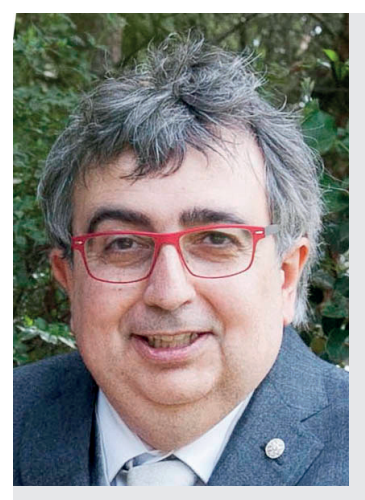

\begin{abstract}
Resumen: Las Information Schools (iSchools) son en la actualidad la red académica de referencia internacional en información. Desde su creación en 2005, unas 80 universidades se han afiliado a la red, a través de las respectivas facultades o departamentos temáticamente involucrados. Las facultades o departamentos académicos que forman parte de las ischools toman como objeto de estudio la información, en estrecha relación con las personas y la tecnología. Se presenta una panorámica general de las ischools que incluye: la historia de sus orígenes y evolución, una revisión actualizada de su actividad académica, y tendencias de futuro. Esta panorámica se traza a través de una revisión crítica de una selección de la bibliografía existente sobre este movimiento académico, que incluye documentos de opinión y debate académico, estudios científicos basados en la recolección y análisis de datos del conjunto de universidades de la red o muestras representativas, y una mirada a la iConference, congreso mundial anual organizado por esta red académica.
\end{abstract}

Palabras clave: Educación, Información; Documentación; Investigación; Docencia; Information Schools; Biblioteconomía; Informática; Ciencias sociales.

Abstract: The Information Schools (iSchools) are a prominent international academic network of information schools. Since its creation in 2005, about 80 universities have joined the network, through the respective faculties or departments thematically involved. The faculties or academic departments that are part of the ischools study information, in close relationship with people and technology. We present a general overview of the ischools that includes: the history of its origins and evolution, an updated review of its academic activity, and future trends. This overview is done through a critical review of a selection of existing literature on this academic movement. The review includes opinion and academic debate documents, scientific studies based on the collection and analysis of data from all the universities in the network or representative samples of them, and a look at the iConference, an annual world congress organized by this academic network.

Keywords: Education; Information science; Research; Teaching; Information Schools; Library science; Computer science; Social sciences. 


\section{Introducción}

Las ischools (Information Schools), como red de referencia internacional en información, inician su andadura oficial en 2005. Ese mismo añotiene lugar también la primera iConference, congreso anual organizado por esta red académica, abierto a la participación de profesionales y académicos de esta área de conocimiento. En la actualidad, a principios de 2018, la red agrupa a 78 facultades y departamentos de universidades en todo el mundo como miembros de pleno derecho (incluidas 3 en España: Universitat Oberta de Catalunya, Universidad Carlos III de Madrid y Universitat Politècnica de València) y 9 más como integrantes asociados (incluye recientemente la Pontificia Universidad Javeriana de Colombia). Todas ellas están interesadas en la información como objeto central de docencia e investigación. Se entiende la información en estrecha interrelación con las personas y la tecnología.

A continuación, resumiremos las raíces y evolución intelectuales de esta red académica hasta el momento presente, trazaremos un panorama de su investigación y docencia, y discutiremos tendencias, retos y escenarios de futuro. Prestaremos atención, por ejemplo, a cómo este movimiento académico ha ido combinando las aportaciones de diversas disciplinas, especialmente biblioteconomía y documentación, e informática.

\section{Orígenes y evolución de la red}

La fundación formal de la red se produce en 2005 por parte de un grupo de 5 facultades: Drexel, Michigan, Penn State, Syracuse y Washington. Entonces tiene lugar la primera iConference, actuando de anfitrión Penn State. El precedente inmediato del inicio oficial de la red de ischools se produce en el curso académico 2002-2003, con la reunión de facultades en North Carolina. Algunas de ellas ya habían empezado a colaborar antes, en 1988. Ese primer germen informal de las iSchools fue establecido por los decanos Toni Carbo (Penn State), Donald Marchand (Syracuse) y Rychard Lytle (Drexel) (Larsen, 2010). Pero veamos con algo más de detalle en qué contexto nace y empieza a desarrollarse este movimiento académico.

\subsection{Los inicios de las iSchools: ideas fuerza y primeros pasos}

En las últimas décadas del siglo pasado, se habían ido produciendo reflexiones sobre la disciplina de library science (Van-House; Sutton, 1996; Williams, 1978), que progresivamente va cambiando su denominación más generalmente aceptada a library and information science. Ese gradual cambio de foco disciplinar, tiene su reflejo en los cambios de nombre de algunas escuelas de especial relevancia, que marcan tendencia. Así:

- 1964: la Graduate Library Schoo/ de la University of Pittsburgh, pasa a llamarse Graduate School of Library and Information Sciences;

- 1968: la University of California Irvine crea el departamento académico de Information and Computer Science;

- 1974, la School of Library Science de Syracuse University pasa a denominarse School of Information Studies.

Un elemento clave para entender esta etapa es el contexto de crisis de los enfoques clásicos de la enseñanza universitaria en biblioteconomía en Norteamérica, que conlleva el cierre de varias titulaciones y facultades, durante la década de 1980 y parte de la década de 1990. Ahora bien, las universidades del grupo fundador van más allá de una mera reacción coyuntural a esa crisis, y plantean, primero a nivel individual y luego de forma concertada, unas reflexiones de fondo respecto a un cambio estructural en el 
estudio académico de la información, ante los escenarios radicalmente nuevos que conlleva la "explosión" de información digital masiva e interconectada. Así, de los posicionamientos de académicos activos en la red estos primeros años cabe destacar una serie de ideas fuerza que inspiran este movimiento académico.

En primer lugar, se plantea la aspiración a una comprensión amplia y profunda del papel que juega la información en las actividades humanas como motor de progreso. Ello conlleva la resolución de cuestiones sobre la naturaleza de la información, sobre su uso social y sobre el diseño adecuado de sistemas, servicios y entornos (Bates, 2010). Esta aspiración comporta varias implicaciones:

- supone plantearse el estudio de la información abstrayéndose de un determinado entorno organizativo o profesional concreto, al efecto de crear conocimiento de aplicación en todos esos entornos;

- otra implicación es un tratamiento interdisciplinar, que sea capaz de combinar los desarrollos teóricos y pragmáticos aportados por diferentes áreas de conocimiento hasta entonces, en relación con información, personas, tecnología y sus interrelaciones.

Teniendo en cuenta todo ello, otras ideas fuerza de este movimiento son:

- alto compromiso con la investigación;

- apuesta por la innovación y el trabajo en red;

- estrecha relación con el entorno industrial y profesional;

compromiso con la excelencia;

- énfasis en la comunicación y el marketing.

Son diversos los artículos en los primeros años de funcionamiento formal de la red donde se exponen y discuten estos posicionamientos y aspiraciones, o se analizan los primeros pasos de su recorrido en la práctica. Se trata casi siempre de artículos firmados por académicos activos de este movimiento, con el consiguiente sesgo favorable (Chu, 2012; Dillon, 2012; Brynko, 2012; Bruce, 2011; Milojevic et al., 2011; Larsen, 2010; Pollack, 2010; Olson; Grudin, 2009; Wobbrock; Ko; Kientz, 2009; Cox; Larsen, 2008; Seadle; Greifeneder, 2007).

Ese espíritu fundacional de las iSchools se refleja en la carta de la organización:

"considera un hecho que se requieren competencias profesionales en todas las formas de información para el progreso en ciencia, negocios, educación y cultura. Estas competencias deben incluir la comprensión de los usos y usuarios de información, la naturaleza de la información en sí misma, así como las tecnologías de la información y sus aplicaciones".

http://www.ischools.org/about/charter

De todo ello cabe remarcar algunas valoraciones. La primera de ellas, estamos ante un movimiento de profundo calado académico, que va mucho más allá, por lo menos en sus intenciones, de un simple cambio de denominación o rebranding. En este sentido, como apunta García-Marco (2009) en la primera nota ThinkEPI dedicada al tema, no se trata de una operación cosmética, sino de una ruptura con la educación tradicional en biblioteconomía y documentación. Pérez-Agüera (2010) en la segunda nota ThinkEPI dedicada a las ischools, saluda apasionadamente este movimiento. Habiendo escuchado a uno de los ideólogos del movimiento, el profesor Gary Marchionini, valora especialmente el foco en la información, abstrayéndose para ello de los diferentes contextos profesionales y organizativos, y se pregunta a qué están esperando para entrar las facultades españolas. Pues bien, hay que esperar a 2013 para los primeros ingresos (Universitat Oberta de Catalunya, Universitat Politècnica de València) y para una primera nota ThinkEPI escrita por un académico como participante en la red (Cobarsí-Morales, 2014). Y hasta 2014 no se produce la entrada de Universidad Carlos III de Madrid.

\section{"El explosivo crecimiento y globalización de esta red académica en tan pocos años constituye por sí sólo un indicador de su impacto"}

Por tanto, en nuestro país, la afiliación entre las facultades con potencial para ello, a priori las facultades de información y documentación y las facultades de informática, no toma ni mucho menos las dimensiones que a día de hoy tiene en Estados Unidos, Reino Unido o la Europa nórdica. Cabe señalar en este sentido que la presencia portuguesa es, proporcionalmente, bastante destacable, con 3 universidades: Universidade do Porto, Universidade Nova de Lisboa y Universidade do Minho.

Tampoco en América del Norte, a pesar de la alta afiliación y de acoger al núcleo fundador, ha habido una aceptación unánime del movimiento iSchools entre el mundo académico. Desde el principio, voces autorizadas dentro de la academia de biblioteconomía y documentación expresan, incluso con cierta acritud, su escepticismo sobre su contenido real (Cronin, 2005). Otros, a menudo 
participantes activos, ven este movimiento como una vía de evolución deseable para las facultades de biblioteconomía y documentación (Dillon, 2012). King (2006), también académico en una iSchool, reconoce no obstante el reto que supone, para una novedad en el mundo académico, adquirir identidad y legitimidad.

“La creciente importancia del concepto de dato en la actividad académica de las ischools pone en cuestión el concepto tradicional de information science, donde se supone por lo general que la información manejada es registrada por parte de personas"

Por otra parte, como hemos comentado, el foco de las iSchools se centra en las interrelaciones en información, tecnología y personas, y ello implica combinar la aportación de diferentes áreas de conocimiento. Olson y Grudin (2009), proponen en especial las siguientes:

- respecto a información: biblioteconomía, archivística y recuperación de información;

- respecto a tecnología: informática;

- respecto a personas: psicología, sociología, antropología y gestión de organizaciones.

Para una discusión monográfica sobre la interrelación de la archivística y el movimiento iSchools, véase el trabajo de Cox y Larsen (2008).

\subsection{Composición de la red y su evolución}

Como acabamos de ver, según plantean Olson y Grudin (2009), en línea con el discurso de las ischools, son unas cuantas las disciplinas científicas concernidas por este movimiento respecto a su potencial aportación. Ahora bien, cuando ellos mismos establecen la disciplina científica dominante en la tradición intelectual de las facultades que conforman la red académica por aquel entonces, apuntan que el grueso procede de Library and Information Science (15), algunas otras de Computer Science (4), y 2 de otras disciplinas. Ello cuadra con la clasificación establecida por Wu et al. (2011) sobre las disciplinas intelectuales de origen de las facultades que en esa fecha integran la red. EI propio autor de este informe ha ido siguiendo la composición de la red a lo largo del tiempo, desde 2013. Considera consistentes las tipificaciones de Olson y Grudin (2009) y Wu et al. (2011), y ha ido aplicando esos mismos criterios a medida que iban apareciendo nuevos integrantes, de manera que podemos disponer de esos datos ordenados cronológicamente y actualizados, tal y como aparecen en la tabla 1 . Se recogen únicamente los miembros de pleno derecho y no se incluyen los asociados, generalmente facultades o escuelas de menor dimensión y sin estudios de doctorado. Para conocer más en detalle las ischools afiliadas en los primeros años (2005-2008), véase el artículo de Larsen (2010).

"A partir de sus orígenes norteamericanos, el aumento de integrantes de la red ha ido paralelo a una clara internacionalización y globalización. Desde 2016, las iSchools norteamericanas son menos que las del resto del mundo"

Cabe remarcar que las facultades de tradición Library \& Information Science son una mayoría clara a lo largo del tiempo, pero con cierta tendencia a decrecer su proporción hasta el actual 59\%. Lo contrario sucede con las facultades procedentes del ámbito de Computer Science, cuya presencia

Tabla 1. Composición de la red iSchools según la tradición temática (número y porcentaje de facultades)

\begin{tabular}{|l|c|c|c|c|c|}
\hline $\begin{array}{c}\text { Tradición } \\
\text { académica de } \\
\text { origen }\end{array}$ & $\begin{array}{c}\text { Integrantes 2009 } \\
\text { (Olson; Grudin, } \\
\text { 2009) }\end{array}$ & $\begin{array}{c}\text { Integrantes } \\
\mathbf{2 0 1 1} \\
\text { (Wu et al., } \\
\mathbf{2 0 1 1 )}\end{array}$ & $\begin{array}{c}\text { Integrantes 2013 } \\
\text { (elaboración } \\
\text { propia) }\end{array}$ & $\begin{array}{c}\text { Integrantes 2016 } \\
\text { (elaboración } \\
\text { propia) }\end{array}$ & $\begin{array}{c}\text { Integrantes 2018 } \\
\text { (elaboración } \\
\text { propia) }\end{array}$ \\
\hline $\begin{array}{l}\text { Library and } \\
\text { Information } \\
\text { Science }\end{array}$ & $15(71 \%)$ & $19(70 \%)$ & $32(62 \%)$ & $45(61 \%)$ & $46(59 \%)$ \\
\hline $\begin{array}{l}\text { Computer } \\
\text { Science }\end{array}$ & $4(19 \%)$ & $4(15 \%)$ & $13(25 \%)$ & $21(28 \%)$ & $22(28 \%)$ \\
\hline $\begin{array}{l}\text { Business } \\
\text { Management }\end{array}$ & $1(5 \%)$ & $3(11 \%)$ & $6(12 \%)$ & $7(13 \%)$ & $8(10 \%)$ \\
\hline Otroso dudosos & $1(5 \%)$ & $1(4 \%)$ & $1(2 \%)$ & $1(1,5 \%)$ & $2(1 \%)$ \\
\hline Total & 21 & 27 & 52 & 74 & 78 \\
\hline
\end{tabular}


va creciendo con el tiempo hasta el actual $28 \%$. Siendo cuantitativamente minoritarias las facultades tecnológicas, queda claro que forman parte del movimiento desde el principio, están en su ADN podríamos decir, no se entendería el movimiento sin su aportación cualitativa y su presencia cuantitativa es creciente. En cambio, las facultades procedentes de Business Management son claramente minoritarias, y la presencia de otras disciplinas como tradición intelectual principal de las facultades integrantes es prácticamente inapreciable. Ello no desmiente la aportación intelectual de otras disciplinas en este movimiento académico, pero da una idea, por lo menos de trazo grueso, de cuáles son las componentes deconocimiento (y de poder si se quiere) principales.

Otra forma de ver la tradición disciplinar sería, no por facultades, sino mediante los perfiles individuales del propio profesorado. Con esta idea, el trabajo de Wiggins y Sawyer (2012), clasifica al profesorado de las iSchools en fecha 2009 en 8 grandes áreas temáticas (tabla 2). Es interesante ver aquí como hay dos áreas principales, y constatar la primacía clara del área de informática, ingeniería electrónica y matemáticas en cuanto a los perfiles individuales del profesorado, incluso en un momento en que como hemos apuntado, la mayoría clara de facultades integrantes tenía una tradición de

\section{Tabla 2. Perfil del profesorado de las iSchools en 2009 según} Wiggins y Sawyer (2012).
Library and Information Science más marcada que en la actualidad.

Por otra parte, la composición de la red según situación geográfica queda recogida en la tabla 3 , utilizando las mismas fuentes que en la tabla 1 , y recogiendo también aquí únicamente los miembros de pleno derecho. Vemos que, a partir de sus orígenes norteamericanos, el aumento de integrantes de la red ha ido paralelo a una clara internacionalización y globalización. En este sentido un punto de inflexión se observa en 2016, cuando por primera vez las ischools norteamericanas son menos que las del resto del mundo. Por lo que respecta a Europa, su cuota de participación ha ido aumentando hasta un tercio del total. Y para el caso de Asia-Pacífico ha ido subiendo hasta un quinto del total. A día de hoy, no obstante, la red apenas si existe en África (una facultad), y no hay presencia de universidades iberoamericanas ni de países en desarrollo tan importantes como la India o Indonesia como afiliados de pleno derecho (la reciente incorporación de la Pontificia Universidad Javeriana de Colombia 
Tabla 3. Composición de la red iSchools por situación geográfica.

\begin{tabular}{|l|c|c|c|c|c|}
\hline $\begin{array}{c}\text { Situación } \\
\text { geográfica } \\
\text { por } \\
\text { continentes }\end{array}$ & $\begin{array}{c}\text { Integrantes 2009 } \\
\text { (Olson; Grudin, } \\
\text { 2009) }\end{array}$ & $\begin{array}{c}\text { Integrantes } \\
\mathbf{2 0 1 1} \\
\text { (Wu et al., } \\
\mathbf{2 0 1 1 )}\end{array}$ & $\begin{array}{c}\text { Integrantes 2013 } \\
\text { (elaboración } \\
\text { propia) }\end{array}$ & $\begin{array}{c}\text { Integrantes 2016 } \\
\text { (elaboración } \\
\text { propia) }\end{array}$ & $\begin{array}{c}\text { Integrantes 2018 } \\
\text { (elaboración propia) }\end{array}$ \\
\hline $\begin{array}{l}\text { América del } \\
\text { Norte }\end{array}$ & $20(95 \%)$ & $22(81 \%)$ & $28(54 \%)$ & $33(45 \%)$ & $36(46 \%)$ \\
\hline Europa (*) & 0 & $3(11 \%)$ & $17(33 \%)$ & $25(34 \%)$ & $26(33 \%)$ \\
\hline Asia-Pacífico & $1(5 \%)$ & $2(7 \%)$ & $7(13 \%)$ & $15(20 \%)$ & $15(19 \%)$ \\
\hline África & 0 & 0 & 0 & $1(1 \%)$ & $1(1 \%)$ \\
\hline Total & 21 & 27 & 52 & 74 & 78 \\
\hline
\end{tabular}

(*) Incluye Turquía e Israel

es como asociado). Para puntos de vista más cualitativos y específicos sobre la situación de las iSchools en los diferentes continentes pueden consultarse diversas entrevistas a profesores con cargos directivos en la red aparecidas en el boletín de ASIS\&T (Larsen, 2016; Seadle, 2016; Oh, 2016) y la nota ThinkEPI de López-Borrull y CobarsíMorales (2017).

Por último, y no menos importante, puede observarse un crecimiento muy rápido en los primeros 9 o 10 años de existencia de la red, que se ha ralentizado algo en lo últimos 2 o 3 años. En todo caso estamos hablando de un número muy importante de facultades de primer nivel (la lista completa puede consultarse en el sitio web ischools.org), que es una muestra de la vitalidad y consolidación de esta red académica, conseguida en muy pocos años.

http://ischools.org/members/ directory

\subsection{Una mirada a la iConference}

Otro indicador de esta creciente globalización y vitalidad de la red nos lo da la iConference, congreso anual organizado por las iSchools, sobre el cual presentamos un resumen de datos de los diez últimos años en la tabla 4.

Como puede observarse en la tabla, en los últimos años la iConference viene celebrándose rotatoriamente en distintos continentes y congrega medio millar de asistentes a proximadamente. Los proceedings de las últimas ediciones pueden consultarse en:

http://ischools.org/theiconference/proceedings
Cabe destacar que a partir de 2018 el texto completo de lospapers aceptados en la iConference se publica en Lecture notes in computer science.

En cada edición de la iConference se otorgan diversos premios, destacando los otorgados a la mejor tesis doctoral y el mejor paper de resultados completos. En la tabla 5 se hace una recopilación de los trabajos premiados desde 2013 (año en que se convoca por primera vez el premio a la mejor tesis). Ello da una cierta idea de los temas de interés prioritarios en los últimos tiempos: social media, visualización de información, políticas de información, ciencia de datos, etc.

Por lo que respecta a la presencia española en la iConference, ha empezado a apuntar en los últimos años, tanto desde el punto de vista de organización como científico. Así, las profesoras ischools

LEADING AND PROMOTING THE INFORMATION FIELD.

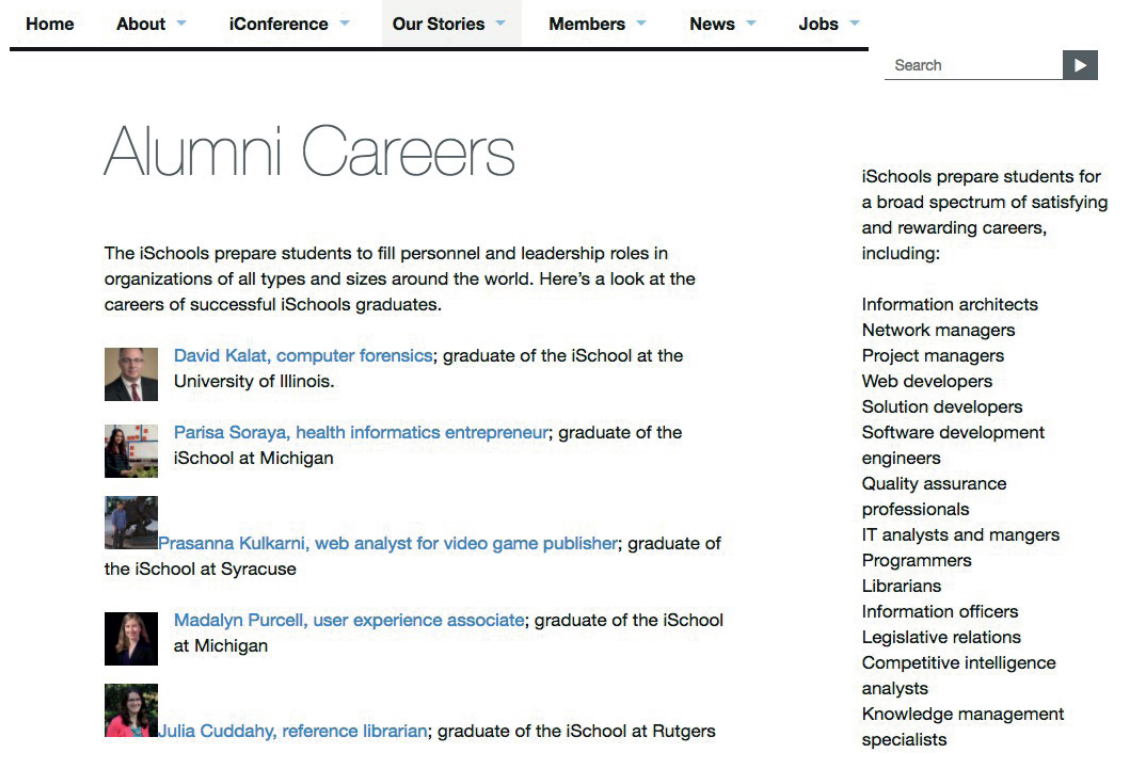

https://ischools.org/stories/careers 
Tabla 4. Cuadro resumen de las iConferences

\begin{tabular}{|c|c|c|c|}
\hline Año & Lugar & Organizadores & $\begin{array}{l}\text { Número de } \\
\text { participantes }\end{array}$ \\
\hline 2018 & $\begin{array}{l}\text { Sheffield } \\
\text { (Reino Unido) }\end{array}$ & $\begin{array}{l}\text { Information School (University of Sheffield, Reino Unido). } \\
\text { Computer and Information Sciences (Northumbria University, Reino Unido). }\end{array}$ & No disponible \\
\hline 2017 & Wuhan (China) & $\begin{array}{l}\text { School of Information Management (Wuhan University, China). } \\
\text { Library and Information Science and Data Science Department (Sungkyunkwan } \\
\text { University, Corea del Sur). }\end{array}$ & 482 \\
\hline 2016 & $\begin{array}{l}\text { Philadelphia } \\
\text { (Estados Unidos) }\end{array}$ & College of Computing and Informatics (Drexel University, Estados Unidos). & 467 \\
\hline 2015 & $\begin{array}{l}\text { Newport Beach, } \\
\text { California } \\
\text { (Estados Unidos) }\end{array}$ & $\begin{array}{l}\text { The Donald Bren School of Information and Computer Sciences (University of } \\
\text { California Irvine, Estados Unidos). }\end{array}$ & 531 \\
\hline 2014 & Berlin (Alemania) & $\begin{array}{l}\text { School of Library and Information Science (Humbold Universitat, Alemania). } \\
\text { Royal School of Library and Information Science (University of Copenhaguen, } \\
\text { Dinamarca). }\end{array}$ & 450 \\
\hline 2013 & $\begin{array}{l}\text { Fort Worth } \\
\text { (Estados Unidos) }\end{array}$ & College of Information (University of North Texas, Estados Unidos). & 512 \\
\hline 2012 & Toronto (Canadá) & Faculty of Information (University of Toronto, Canadá). & 486 \\
\hline 2011 & $\begin{array}{l}\text { Seattle } \\
\text { (Estados Unidos) }\end{array}$ & Information School (University of Washington, Estados Unidos). & 538 \\
\hline 2010 & $\begin{array}{l}\text { Illinois } \\
\text { (Estados Unidos) }\end{array}$ & $\begin{array}{l}\text { Graduate School of Library and Information Studies (University of Illinois at } \\
\text { Urbana-Champaign, Estados Unidos). }\end{array}$ & 346 \\
\hline 2009 & $\begin{array}{l}\text { Chapell Hill } \\
\text { (Estados Unidos) }\end{array}$ & $\begin{array}{l}\text { School of Information and Library Science (University of North Carolina at } \\
\text { Chapell Hill, Estados Unidos). }\end{array}$ & 305 \\
\hline 2008 & $\begin{array}{l}\text { Los Ángeles } \\
\text { (Estados Unidos) }\end{array}$ & $\begin{array}{l}\text { Graduate School of Education and Information Studies (University of California } \\
\text { Los Ángeles, Estados Unidos). }\end{array}$ & 277 \\
\hline
\end{tabular}

Elaboración propia según datos de http://www.ischools.org

Virginia Ortiz-Repiso y Eva Méndez (Universidad Carlos III de Madrid) participan en la organización de la iConference 2016 de Philadelphia como Early Career Colloquium Chair y Conference Communications Director respectivamente.

En el Programme Committee de Wu-Han 2017 participan Virginia Ortiz-Repiso (UC3M), Josep Cobarsí-Morales (UOC) y Eduardo Vendrell (UPV). También en Wu-Han, Virginia Ortiz-Repiso coorganiza el workshop "Information science to data science: New directions".

En la iConference de Sheffield 2018 diversos profesores intervinieron como reviewers: Josep Cobarsí-Morales, Núria Ferran-Ferrer y Mercè Vázquez-García (UOC), Virginia Ortiz-Repiso (UC3M) y Antonia Ferrer Sapena (UPV).

En Sheffield también se defiende el primer paper elaborado por académicos de una

Tabla 5. Cuadro resumen de trabajos científicos premiados en las últimas iConferences

\begin{tabular}{|l|l|l|}
\hline Año & \multicolumn{1}{|c|}{$\begin{array}{c}\text { Título de la tesis doctoral ganadora del premio } \\
\text { anual }\end{array}$} & \multicolumn{1}{|c|}{$\begin{array}{c}\text { Título del paper de resultados completos ganador } \\
\text { del premio anual }\end{array}$} \\
\hline 2018 & Emotion in social media & $\begin{array}{l}\text { Understanding interactions between municipal police } \\
\text { departments and the public on Twitter }\end{array}$ \\
\hline 2017 & $\begin{array}{l}\text { It's not Rocket Library Science: Design epistemology and } \\
\text { American librarianship }\end{array}$ & $\begin{array}{l}\text { An in-depth analysis of tags and controlled metadata for } \\
\text { book search }\end{array}$ \\
\hline 2016 & $\begin{array}{l}\text { Where in the world is Internet? Locating political power } \\
\text { in internet infrastructure }\end{array}$ & $\begin{array}{l}\text { The work of reuse: Birth certificate data and healthcare } \\
\text { accountability measurements }\end{array}$ \\
\hline 2014 & $\begin{array}{l}\text { Factors that influence adoption an use of location- } \\
\text { sharing social media }\end{array}$ & $\begin{array}{l}\text { "Shesaid yes!"-Liminalityand engagementannouncements } \\
\text { on Twitter } \\
\text { representation of personal archives }\end{array}$ \\
\hline 2013 & $\begin{array}{l}\text { Image-enabled discourse: Investigating the creation of } \\
\text { visual information as communicative practice }\end{array}$ & $\begin{array}{l}\text { Developing design interventions for cyberbullying: A } \\
\text { narrative-based participatory approach }\end{array}$ \\
\hline
\end{tabular}

Elaboración propia según datos de http://www.ischools.org 


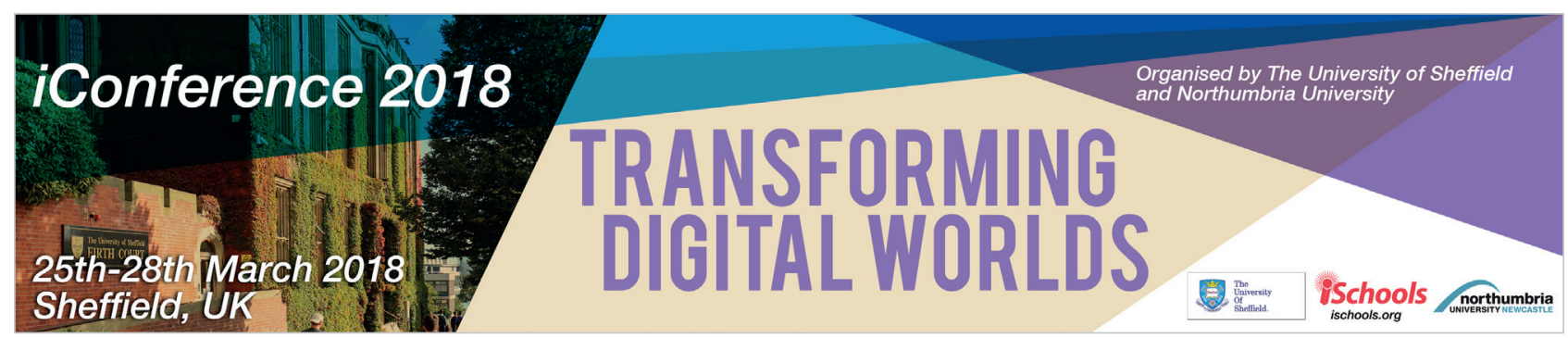

https://ischools.org/the-iconference/call-for-participation

universidad española (Cobarsí-Morales; Canals, 2018). Se ha iniciado, por tanto, la participación efectiva de nuestro país en la iConference en los años recientes, quedando no obstante un largo camino por recorrer.

\section{Panorama actual}

En la tabla 6 se recoge una selección de los trabajos científicos publicados recientemente, desde 2016 a la actualidad, sobre la docencia y/o sobre la investigación en las ischools. Su revisión nos permitirá hacernos una idea del estado y evolución de su actividad académica. Se han escogido los trabajos que incluyen la recogida y análisis de datos para el conjunto de facultades de la red ischools, o bien para una muestra amplia o representativa de estas, y no se han tenido en cuenta los estudios de caso de instituciones individuales, o aquellos que presentaban resultados muy preliminares.

Una de las características que presenta la red en la actualidad es una importante presencia del concepto de dato y materias relacionadas en sentido amplio (digital curation, data science, big data) en las iSchools. Ello queda de manifiesto en el trabajo más reciente sobre la red (OrtizRepiso; Greenberg; Calzada-Prado, 2018): aproximadamente dos tercios de las facultades ofrecen algún tipo de titulación relacionada con datos, y un $15 \%$ de la oferta de titulaciones observada se centra en el concepto de dato. Estos resultados son coherentes con los de Kang y Moon (2017), quiénes con criterios algo más laxos consideran que la práctica totalidad de las facultades ofrece algún curso sobre datos, y que el $17 \%$ de portfolio se focaliza en el concepto de dato. Además constatan la notable presencia de cursos donde se vincula el concepto de dato con health y con management. Por tanto, podemos considerar que la "datificación" es una tendencia de la red, ya apuntada también anteriormente por Li et al. (2013).

En cambio, el concepto de sostenibilidad vinculado con información apenas si tiene presencia. Según Chowdhury y Koya (2017), solamente 13 de las facultades ofrecen cursos relacionados con ese concepto.

Una cuestión que podemos plantearnos es hasta qué punto las iSchools, procediendo de distintas tradiciones académicas, convergen entre ellas. El estudio de Zuo, Zhao y Eichmann (2016) viene a dar una respuesta a esto, aunque sea limitada a las ischools de América del Norte. Constatan la existencia de una red considerable de reclutamiento de académicos entre estas facultades. Por lo que respecta al tipo de doctorado del profesorado, ganan peso los que ha realizado un doctorado en información (entendido como tal information studies, information transfer, informatics) casi un $27 \%$ del profesorado junior, en detrimento de doctorados en biblioteconomía (entendido como tal library, information and library science) que supone solamente un $12 \%$ del profesorado junior, y con un peso dominante en

Tabla 6. Trabajos científicos recientes que analizan las iSchools

\begin{tabular}{|c|l|l|l|}
\hline Año & \multicolumn{1}{|c|}{ Autoría } & \multicolumn{1}{|c|}{ Objeto de estudio } & \multicolumn{1}{|c|}{ Población estudiada } \\
\hline 2018 & $\begin{array}{l}\text { Ortiz-Repiso, } \\
\text { Greenberg y Calzada- } \\
\text { Prado }\end{array}$ & $\begin{array}{l}\text { Datos como materia en el curriculum académico } \\
\text { de las iSchools }\end{array}$ & El conjunto de las iSchools \\
\hline 2017 & Chowdhury y Koya & $\begin{array}{l}\text { Sostenibilidad de la información en las iSchools: } \\
\text { docencia e investigación }\end{array}$ & El conjunto de las iSchools \\
\hline 2017 & Kang y Moon & $\begin{array}{l}\text { Ciencia de datos en las iSchools y su conexión } \\
\text { con otras disciplinas }\end{array}$ & El conjunto de las iSchools \\
\hline 2016 & Burton & $\begin{array}{l}\text { Análisis de los cursos mediante las referencias } \\
\text { bibliográficas de los programas }\end{array}$ & $\begin{array}{l}\text { Subconjunto de las iSchools: las 24 que } \\
\text { forman parte del iCaucus }\end{array}$ \\
\hline 2016 & Zuo, Zhao y Eichmann & $\begin{array}{l}\text { Background del profesorado y producción } \\
\text { científica en las iSchools }\end{array}$ & Las iSchools en Estados Unidos \\
\hline
\end{tabular}


Tabla 7. Temas clave de investigación según Zuo, Zhao y Eichmann (2016)

\begin{tabular}{|c|c|}
\hline Temas principales (en 2014) & Temas que han ido subiendo constantemente desde 1988 \\
\hline $\begin{array}{l}\text { Tecnologías de la información para la colaboración y la } \\
\text { comunicación }\end{array}$ & Redes sociales y social media \\
\hline Ingeniería de software y de sistemas & $\begin{array}{l}\text { Tecnologías de la información para la colaboración y la } \\
\text { comunicación }\end{array}$ \\
\hline Privacidad de la información y políticas de la información & Recuperación de información y personalización \\
\hline Redes sociales y social media & Almacenamiento y visualización de datos \\
\hline Machine learning y minería de datos & Interfaz y experiencia de usuario \\
\hline Recuperación de información y personalización & Health informatics \\
\hline Infraestructura informática & Bioinformática \\
\hline
\end{tabular}

el profesorado junior de profesores procedentes de doctorados en ciencias de la computación (considerando aquí como computer science, electrical engineering, mathematics, computer engineering) que supone más del $31 \%$. Esta composición del profesorado podría sorprender si tenemos en cuenta las tradiciones disciplinares de origen apuntadas en la tabla 1, y viene a indicar que las iSchools han tenido (por lo menos en Norteamérica) una evolución notable respecto a la composición de su profesorado. Esta evolución es coherente con el hecho de centrarse en la relación entre personas, información y tecnología, y con la tendencia a la 'datificación'. Sería interesante conocer la situación en Europa y Asia-Pacífico, donde el movimiento de afiliación de facultades es mucho más reciente, pero no resulta fácil intuir los resultados.

Este mismo estudio de Zuo, Zhao y Eichmann (2016) se plantea también cuáles son los temas dominantes de investigación de las ischools en América del Norte. En la tabla 7 se recogen los 7 temas más prevalentes en tiempos recientes y los 7 temas que han ido creciendo constantemente desde finales de la década de los años 80 . También aquí se desconoce la situación en otros continentes, pero se podría aventurar que un estudio similar respecto al conjunto de la red arrojaría resultados parecidos. De hecho, los resultados del estudio anterior de Holmberg, Tsou y Sugimoto (2013), realizado en fecha anterior sobre el conjunto de las iSchools, guardan bastante coherencia con Zuo, Zhao y Eichmann (2016).

El trabajo de Burton (2016) representa una primera aproximación a los conceptos clave de docencia de las iSchools y se realiza sobre un subconjunto de 24 iSchools, las que conforman el iCaucus. La lista de las revistas más citadas en los programas de los cursos (tabla 8) permite constatar el peso de Library and Information Science. Pero la presencia en esta lista de Harvard Business Review e Interactions, así como el destacado primer lugar de Journal of the Association for
Information Science and Technology, permiten poner de manifiesto la interdisciplinariedad. No obstante, los autores constatan la dificultad de encontrar referencias bibliográficas concretas y conceptos susceptibles de constituir un core en el curriculum docente. En este sentido, no aportan mucho más a esta cuestión desde el punto de vista empírico que la primera aproximación realizada hace mucho tiempo por Seadle y Greifeneder (2007).

Otros artículos recientes no recogidos en la selección de la tabla 6 , presentan resultados muy fragmentarios y preliminares sobre facetas de la actividad académica de las ischools, tales como:

- acciones de engagement a los estudiantes (Lieutenant; Kules, 2016);

- el potencial de investigación sobre archivística en las iSchools (Leazer, 2016),

- comparativa intercultural entre el curriculum de master en China y en Estados Unidos (Kowalczyk; Gao, 2017),

- tesis doctorales e interdisciplinariedad (Xie; Zhou; Fan, 2017; Beaton; Jeng; Champagne, 2014),

- las diferencias temáticas entre iSchools de tradición bibliotecaria y el resto de facultades de biblioteconomía en Estados Unidos (Shu; Beheshti, 2016).

Tabla 8. Revistas más citadas en los programas de asignaturas de una muestra de 24 iSchools según Burton (2016)

\begin{tabular}{|c|l|}
\hline Ranking & \multicolumn{1}{c|}{ Revista } \\
\hline 1 & $\begin{array}{l}\text { Journal of the American Society for Information } \\
\text { Science \& Technology }\end{array}$ \\
\hline 2 & Journal of documentation \\
\hline 3 & Information processing \& management \\
\hline 4 & Library trends \\
\hline 5 & College \& research libraries \\
\hline 6 & Harvard business review \\
\hline 7 & Interactions \\
\hline
\end{tabular}


Merecen una mención en tanto que esas facetas podrían estudiarse con más profundidad en futuros trabajos sobre esta red académica.

\section{Conclusiones, incógnitas y futuribles}

Recapitulando lo visto hasta ahora, podemos plantearnos algunas conclusiones sobre el estado y tendencias de las ischools. Y también podemos constatar los límites del conocimiento disponible sobre esta red académica, susceptibles de ser objeto de futuros trabajos de investigación. Asimismo, podemos plantearnos algunos futuribles sobre este movimiento.

\section{“Las universidades españolas pioneras en la red son Universitat Oberta de Catalunya y Universitat Politècnica de València, que ingresan en 2013, seguidas en 2014 por la Universidad Carlos III de Madrid"}

Por lo que respecta a lo que sabemos de la actualidad y tendencias, cabe señalar:

- iSchools haberlas haylas y han venido para quedarse. El explosivo crecimiento y globalización de esta red académica en tan pocos años constituye por sí sólo un indicador de su impacto, por lo menos cuantitativo. La entrada de tantas universidades en tan poco tiempo, conlleva una serie de cuestiones y controversias en cuanto a la gobernanza y cohesión de la red, entre viejos y nuevos integrantes, de tradiciones disciplinares distintas y de países diversos. En tanto que participante, el autor de este informe ha podido observarlo y vivirlo. Sin embargo, no me consta ninguna baja efectiva de instituciones en una red tan amplia ni preveo que pueda haberlas en un futuro cercano.

- Desde un punto de vista cualitativo, puede considerarse, con Budd (2015), que la red ha supuesto una aceleración en los procesos de cambio que vienen dándose en el mundo académico de información y documentación desde hace décadas, y un aumento de la producción científica. El trabajo de Zuo, Zhao y Eichmann (2016) apunta a que, por lo menos en Estados Unidos, la red de reclutamiento de académicos entre ellas se ha reforzado considerablemente y se tiende a un background común del profesorado donde las tecnologías de la información tienen un gran peso, dentro de un contexto donde se combinan varias disciplinas. También son identificables unos temas de interés en cuanto a investigación que reflejan las ideas fundacionales sobre información, personas y tecnología.

- La participación española en la red, iniciada con el ingreso en 2013 de Universitat Oberta de Catalunya y la Universitat Politècnica de València, y en 2014 de la Universidad Carlos III de Madrid, ha empezado a notarse en las últimas iConferences, pero queda un largo camino por recorrer.

- La creciente importancia del concepto de dato en la actividad académica de las iSchools (Ortiz-Repiso; Greenberg; Calzada-Prado, 2018; Kang; Moon, 2017). En este sentido, apuntamos con Chowdhury y Koya (2017) la importancia cualitativa de este hecho, pues el big data pone en cuestión el concepto tradicional de information science donde se supone por lo general que la información manejada es registrada por parte de personas.

En cuanto a ciertos déficits de conocimiento sobre la red, podemos destacar lo siguiente:

- No existe a día de hoy una visión completa y actualizada de la docencia en las Information Schools, los trabajos existentes o bien se refieren a temas de docencia específicos como los datos (Ortiz-Repiso; Greenberg; Calzada-Prado, 2018) o bien han quedado temporalmente obsoletos, como el de Chu (2012).

- También falta una visión global de los temas de investigación y de las redes de reclutamiento de profesorado, refiriéndose el trabajo de Zuo, Zhao y Eichmann (2017) exclusivamente a Estados Unidos y habiendo interesantes referencias previas como Holmberg, Tsou y Sugimoto (2013) y Wiggins y Sawyer (2012). Por otra parte, podrían considerarse aspectos más específicos, en la línea de complementar o continuar trabajos como los apuntados al final de la sección 3.

Finalmente, podemos apuntar algunos futuribles. En este sentido, parece que hay diversos temas clave pendientes para la red a nivel global, a destacar los siguientes:

- La cuestión de cómo articular una colaboración y engagement efectivo de las instituciones y del profesorado y estudiantes, más allá de lo ya conseguido sobretodo en el ámbito geográfico de Estados Unidos.

- Avanzar en una real interdisciplinariedad, siendo ello clave para cuestiones como el tratamiento en docencia e investigación del concepto de dato (Lyon; Brenner, 2015). O para potenciar el tratamiento académico de la sostenibilidad vinculado a información, todavía incipiente como se muestra en Chowdhury y Koya (2017). 
- Avanzar en la definición de unos elementos core del curriculum docente, cuestión apuntada por primera vez por Seadle y Greifeneder (2007) y largamente pendiente.

Por otra parte, y a un nivel más local en nuestro país, hay un largo camino por recorrer. Tanto a nivel del número de instituciones presentes, como de la participación individual del profesorado en la iConference, hay potencial para tener una presencia creciente, a partir de los primeros pasos dados desde 2013 y 2014 por la Universitat Oberta de Catalunya, Universitat Politècnica de València y Universidad Carlos III de Madrid. En este sentido cabe animar a las facultades de información y documentación y a las facultades politécnicas españolas a plantearse el ingreso. Y animar también a los académicos españoles a plantearse la participación a título individual en la iConference, congreso anual abierto a la participación de académicos de cualquier institución, sea afiliada o no a las ischools.

\section{Referencias}

Bates, Marcia (2010). An operational definition of the information disciplines.

https://pages.gseis.ucla.edu/faculty/bates/articles/pdf/ Contribution512-1.pdf

Beaton, Brian; Jeng, Wei; Champagne, Ryan (2014). "Exploring the Use of 'Acknowledgement analysis' to map intellectual diversity and cross-disciplinary activity within the iSchools". En: iConference 2014 Proceedings, pp. 700-707.

https://doi.org/10.9776/14330

Bruce, Harry (2011). "The audacious vision of Information Schools". Journal of library and information science (Taipei), v. 37, n. 1, pp. 4-10.

Brynko, Barbara (2012). "iSchools: shaping the Information Landscape". Information today, n. 29, pp. 1-35.

Budd, John M. (2015). "Productivity of US LIS and iSchool Faculty". Library \& information sesearch, n. 37, pp. 290-295.

https://doi.org/10.1016/j.lisr.2015.11.001

Burton, Matt (2016). “Looking for the core: Preliminary explorations of iCaucus Syllabi". En: iConferece 2016 Proceedings.

https://doi.org/10.9776/16225

Chowdhury, Gobinda; Koya, Kushwanth (2017). "Information practices for sustainability: Role of iSchools in achieving the UN sustainable development goals (SDGs)". Journal of the Association for Information Science and Technology, v. 68, n. 9, pp. 2128-2138. https://doi.org/10.1002/asi.23825

Chu, Heting (2012). "iSchools and non-iSchools in the USA: An examination of their master's programs". Education for information, n. 29, pp. 1-17.

https://doi.org/10.3233/EFI-2010-0908
Cobarsí-Morales, Josep (2014). "Information Schools. Perspectiva y tendencias en 2013". Anuario ThinkEPI, v. 8, pp. 52-55.

https://recyt.fecyt.es/index.php/ThinkEPI/article/ view/29552

Cobarsí-Morales, Josep; Canals, Agustí (2018) "Information and knowledge based conceptual study of 2008 financial crisis". En: Chowdhury, Gobinda; McLeod, Julie; Gillet, Val; Willett, Peter (eds.) Transforming Digital Worlds. iConference 2018. Lecture Notes in Computer Science, v. 10766. Springer, Cham.

https://doi.org/10.1007/978-3-319-78105-1_30

Cox, Richard, J.; Larsen, Ronald L. (2008). "iSchools and archival studies". Archival science, v. 8, n. 4, pp. 307326.

https://doi.org/10.1007/s10502-009-9092-3

Cronin, Blaise (2005). "An I-dentity crisis? The information schools movement". International journal of information management, v.25, n. 4, pp. 363-365. https://doi.org/10.1016/j.ijinfomgt.2005.04.007

Dillon, Andrew (2012). "What it means to be an ischool". Journal of education for library and information science, v. 53, n. 4, pp. 267-273.

https://www. ischool.utexas.edu/ adillon/Journals/ JELIS2012.pdf

García-Marco, Francisco-Javier (2009). "El movimiento iSchools: posicionando los estudios de biblioteconomía y documentación en la era de la información". Anuario ThinkEPI, v. 3, pp. 95-99. http://recyt.fecyt.es/index.php/ThinkEPI/article/ view/32000

Holmberg, Kim; Tsou, Andrew; Sugimoto, Cassidy R. (2013). "The conceptual landscape of iSchools: examining current research interests of faculty members". Information research, v. 18, n. 3.

http://www.informationr.net/ir/18-3/colis/paperC32.htm/

Kang, Ji-Hey; Moon, Eunhye (2017). "Interoperable data science at iSchools. How do iSchools expand their horizons?". En: iConference 2017 Proceedings, pp. 843847.

https://www.ideals.illinois.edu/handle/2142/96734

King, John L. (2006). "Identity in the I-school movement". Bulletin of the American Society for Information Science and Technology, v. 32, n. 4, pp. 13-15.

https://doi.org/10.1002/bult.2006.1720320406

Kowalczyk, Stacy T.; Gao, Yijun (2017). "Information studies in two cultures: A review of iSchool curricula in the China and US". En: iConference 2017 Proceedings, pp. 577-585.

https://www.ideals.illinois.edu/handle/2142/96769

Larsen, Ronald L. (2010). "iSchools". En: Bates, Marcia J.; Maack, Mary N. (eds.). Encyclopedia of Library and Information Sciences, 3rd Edition. New York: Taylor and Francis. ISBN: 9780849397110

Larsen, Ronald L. (2016). "Overview of the iSchool movement. An interview with Ronald L. Larsen, iCaucus Chair". Bulletin of the Association for Information 
Science and Technology, v. 42, n. 4, pp. 12-16. https://onlinelibrary.wiley.com/doi/pdf/10.1002/ bul2.2016.1720420405

Leazer, Gregory (2016). “iSchools, legitimacy, and the contribution of archival studies to the ischool movement". En: iConference 2016 Proceedings, pp. 1-11. https://doi.org/10.9776/16263

Lieutenant, Elizabeth; Kules, Bill (2016). "Are iSchools more adaptable than library schools? Analysis of LIS student engagement in programmatic changes and improvements". En: iConference 2016 Proceedings, pp. 1-4.

http://dx.doi.org/10.9776/16539

López-Borrull, Alexandre; Cobarsí-Morales, Josep (2017). "Information Schools: estado actual, tendencias y propuestas". Anuario ThinkEPI, v. 11, pp. 53-57. https://doi.org/10.3145/thinkepi.2017.05

Lyon, Liz; Brenner, Aaron (2015). "Bridging the data talent gap: Positioning the ischool as an agent for change". International journal of digital curation, v. 10, n. 1, pp. 111-122.

https://doi.org/10.2218/ijdc.v10i1.349

Milojevic, Stasa; Sugimoto, Cassidy R.; Yan, Erjia; Ding, Ying (2011). "The cognitive structure of library and information science: Analysis of article title words". Journal of the American Society of Information Science and Technology, v. 62, n. 10, pp. 1933-1953.

http://dx.doi.org/10.1002/asi.21602

Oh, Sam (2016). "The Asia-Pacific iSchools". Bulletin of the Association for Information Science and Technology, v. 42, n. 4, pp. 26-30.

https://goo.gl/qD1zs2

Olson, Gary M.; Grudin, Jonathan (2009). "The information school phenomenon". Interactions, v. 16, n. 2, pp. 15-19.

http://dx.doi.org/10.1145/1487632.1487636

Ortiz-Repiso, Virginia; Greenberg, Jane; CalzadaPrado, Javier (2018). "A cross-institutional analysis of data-related curricula in information science programmes: A focused look ant the iSchools". Journal of Information Science, v. 44, n. 1, pp. 1-17. https://doi.org/10.1177/0165551517748149

Pérez-Agüera, José-Ramón (2010). “Más sobre iSchools". Anuario ThinkEPI, v. 4, pp. 53-56.

https://recyt.fecyt.es/index.php/ThinkEPI/article/ view/31236/16587

Pollack, Martha E. (2010). "Reflections on the future of the iSchools from a dean inspired by some junior faculty". Interactions, v. 17, n. 1, pp. 66-68.

https://doi.org/10.1145/1649475.1649492

Seadle, Michael (2016). "The European iSchools". Bulletin of the Association for Information Science and Technology, v. 42, n. 4, pp. 26-30. https://goo.gl/neqkSW

Seadle, Michael; Greifeneder, Elke (2007). “Envisioning and ischool curriculum". Information research, v. 12, n. 4. http://www.informationr.net/ir/12-4/colis/colise02.html

Shu, Fei; Beheshti, Jamshid (2016). "The landscape of LIS research and teaching area: iSchools versus non-iSchools in North America". En: iConference Proceedings 2016.

https://goo.gl/VXFbhU

Si, Li; Zhuang, Xiaozhe; Xing, Wenming; Guo, Weining (2013). "The cultivation of scientific data specialists: Development of LIS education oriented to e-science service requirements". Library hi tech, v. 31, n. 4, pp. 700-724.

http://dx.doi.org/10.1108/LHT-06-2013-0070

Van-House, Nancy A.; Sutton, Stuart A. (1996). "The panda syndrome: an ecology of LIS education". Journal of library and information science, v. 37, n. 2, pp. 131-147. http://people.ischool.berkeley.edu/ vanhouse/panda.html

Williams, James G. (1978). "Information science education". En: Brenner, Everett H. (comp.). Information Age in Perspective. Proceedings of the ASIS Annual meeting, New York, 13-17 Noviembre, v. 15. White Plains, N.Y.: Knowledge Industry Publications, pp. 353-357.

Wiggins, Andrea; Sawyer, Steven (2012). "Intellectual diversity and the faculty composition of the iSchools". Journal of the American Society for Information Science and Technology, v. 63, n. 1, pp. 8-21.

https://doi.org/10.1002/asi.21619

Wobbrock, Jacob O.; Ko, Andrew J.; Kientz, Julie A. (2009). "Reflections on the future of ischools from inspired junior faculty". Interactions, v. 16, n.4, pp. 69-71. https://doi.org/10.1145/1572626.1572641

Wu, Dan; He, Daqing; Jiang, Jiepu; Dong, Wuyi; Thien-Wo, Kim (2011). "The state of ischools: an analysis of academic research and graduate education". Journal of information science, v. 38, n. 1, pp. 15-36. https://doi.org/10.1177/0165551511426247

Xie, Sherry L.; Zhou, Wenhong; Fan, Guanyan (2017). "The role of thesis in building information professionals". En: iConference 2017 Proceedings, pp. 598-604.

https://www.ideals.illinois.edu/handle/2142/96771

Zuo, Zhiya; Zhao, Kang; Eichmann, David (2016). "The state and evolution of U.S. iSchools: From talent acquisitions to research outcome". Journal of the Association for Information Science and Technology, v. 68, n. 5, pp. 1266-1277.

http://dx.doi.org/10.1002/asi.23751

Josep Cobarsí-Morales Universitat Oberta de Catalunya Estudios de Informática, Multimedia y Telecomunicación jcobarsi@uoc.edu 